\title{
Fabrication of Nanostructured Polyamic Acid Membranes for Antimicrobially Enhanced Water Purification
}

\author{
Ibrahim Kimotho, ${ }^{1}$ Naumih M. Noah $\mathbb{D}^{0},{ }^{2}$ Mildred Nawiri, ${ }^{1}$ and Betty Mbatia ${ }^{2}$ \\ ${ }^{1}$ Chemistry Department, Kenyatta University, P.O. Box 43844-00100, Nairobi, Kenya \\ ${ }^{2}$ School of Pharmacy and Health Sciences, United States International University-Africa, P.O. Box 14634-00800, Nairobi, Kenya \\ Correspondence should be addressed to Naumih M. Noah; mnoah@usiu.ac.ke
}

Received 16 December 2019; Revised 8 January 2020; Accepted 20 January 2020; Published 17 February 2020

Guest Editor: Guocheng Zhu

Copyright (c) 2020 Ibrahim Kimotho et al. This is an open access article distributed under the Creative Commons Attribution License, which permits unrestricted use, distribution, and reproduction in any medium, provided the original work is properly cited.

\begin{abstract}
Water scarcity and quality challenges facing the world can be alleviated by Point-of-Use filtration devices (POU). The use of filtration membranes in POU devices has been limited largely because of membrane fouling, which occurs when suspended solids, microbes, and organic materials are deposited on the surface of filtration membranes significantly decreasing the membrane lifespan, thereby increasing operation costs. There is need therefore to develop filtration membranes that are devoid of these challenges. In this work, nanotechnology was used to fabricate nanostructured polyamic acid (nPAA) membranes, which can be used for microbial decontamination of water. The PAA was used as support and reducing agent to introduce silver nanoparticles (AgNPs) and gold nanoparticles (AuNPs) with antimicrobial properties. The nPAA membranes were fabricated via thermal and wet phase inversion technique and then tested against Escherichia coli and Staphylococcus aureus following standard tests. The resulting nanoparticles exhibited excellent dispersibility and stability as indicated by the color change of the solution and increments of optical density at $415 \mathrm{~nm}$ for AgNPs and $520 \mathrm{~nm}$ for AuNPs. The wet phase inversion process used produced highly porous, strong, and flexible nPAA membranes, which showed well-dispersed spherical AuNPs and AgNPs whose rough average size was found to be $35 \mathrm{~nm}$ and $25 \mathrm{~nm}$, respectively. The AgNPs demonstrated inhibition for both gram positive E. coli and gram negative $S$. aureus, with a better inhibitory activity against $S$. aureus. A synergistic enhancement of AgNPs antimicrobial activity upon AuNPs addition was demonstrated. The nPAA membranes can thus be used to remove microbials from water and can hence be used in water purification.
\end{abstract}

\section{Introduction}

Water, as a source of life, is the most important material for human survival and development [1]. Even though $71 \%$ of the earth's surface is covered with freshwater resources that can be directly used by humans, about 1.2 billion people have inadequate access to fresh potable water [2]. This is due to rapid development of industries and increasing human activities, such as metal plating, fertilizers, tanneries, mining, paper, batteries, pesticides, and many harmful inorganic and organic pollutants that are released into water $[3,4]$, which seriously endangers the freshwater resource and ecological environment, which undermines the water quality making it unsafe [1]. Diseases from unsafe drinking water and lack of basic sanitation kill more people every year than all forms of violence, accidents, and other diseases. Globally, World Health Organization (WHO) reports that 1 in 8 persons lacks access to safe drinking water and estimates that 3.5 million people, of which $84 \%$ are children and $98 \%$ occur in the developing countries, die every year from waterborne diseases emanating from drinking unsafe water $[2,5,6]$. The need to provide safe drinking water especially to poor people in developing countries cannot be overemphasized [7]. The Increasing global need for water and wastewater treatment has driven the widespread development of large-scale membrane filtration processes [8]. However, the seemingly unavoidable occurrence of filtration membrane fouling is a problem throughout the water treatment industry [8]. Membrane fouling arises when microbes, suspended solids, and organic material are deposited on the surface of 
membranes during selective water permeation [8]. Recently, increasing water demands have forced research into membranes with a superior water flux. With this progress in water flux enhancement, research into membrane antifouling has become more and more pressing as membrane fouling worsens along with increasing water flux $[9,10]$. Fouled membranes have substantially shorter lifespan and lessened filtration efficiency. Reverse osmosis (RO) and forward osmosis (FO) membranes depict the highest fouling contrasting nano-, ultra-, and microfiltration membranes because of their small pore sizes. In RO, ions, unwanted molecules, and larger particles are removed from water by passing them through a semipermeable membrane, while in FO there is a spontaneous diffusion of water across the semipermeable membrane in response to a difference in solute concentration. These membranes also require increasingly high pressure operations to boost water flux and are expensive to maintain and operate $[11,12]$.

Nanotechnology, the science and art of manipulating matter at the atomic and molecular level have the potential to deliver affordable and effective solutions for this problem in water sanitation/purification, providing access to safe drinking water to millions of people [13] and therefore contributing to poverty alleviation and achievement of the sustainable development goals (SDGs). Due to their rapid advancement, nanotechnology and production of advanced nanomaterials offer significant opportunities for extensive range of application for detection and remediation of broad range of environmental contaminants [14]. The integration of polymeric materials with synthetic nanomaterials may permit unprecedented ability to detect, disinfect, and completely remove pathogens in water [15]. Nanoparticles' insertion onto polymeric materials continues to appeal as a suitable process since it leads to versatile materials of diverse applications $[14,16]$ since they have large surface to volume ratio; thus, surface related phenomena and properties are drastically affected with slight modification of size, shape, and surrounding media [17]. Consequently, the desired electronic, catalytic, and optical properties of nanoparticles, depending on the application, can be tuned by generating nanoparticles of defined size and shape in selected media. This allows for the development of new effective nanomaterials and nanodevices [14, 16-20]. Gold nanoparticles, for instance, show very high chemical reactivity compared to bulk gold, well-known for being inert. This kind of nanoparticles has multiple applications in biosensing, gene transfer, as bioprobes in cells and as labels for biomolecules during nano- and microscale visualization, for drug delivery, to enhance electroluminescence and quantum efficiency in organic light emitting diodes [21]. Polymeric materials provide an excellent dispersion and sequestering environment for nanoparticles [22]. Water and waste-water treatment in particular have harnessed the potential of nanoassisted polymeric materials to defeat fouling and biofouling $[20,23]$. Surface modification of polyaniline, polysulfone, and cellulose acetate, among others has been done with nanoparticles to make better membrane materials $[24,25]$. Most of these polymers have the character to act as reductants and stabilizers of metal nanoparticles
[26, 27]. However, their application is limited due to low processability, insolubility in a wide range of solvents, and non-biodegradability [26]. Polyamic acid (PAA), the highly reactive intermediate of polyimides, is soluble in both organic and aqueous solvents, demonstrates good mechanical stability, and acts as a better redox polymer than previously employed polymeric materials $[28,29]$.

In this work, we have incorporated gold and silver nanoparticles into a polyamic acid framework to fabricated antimicrobially enhanced nanostructured conducting pol$\mathrm{y}$ (amic) acid (nPAA) membranes, which can be used as water treatment materials. Embedding the nanoparticles in the PAA membranes changes their properties, making them hydrophilic, or water-attracting, so that water passes through more easily. The ultimate aim of this work is to make low cost nanofilter cartridges using the nPAA membranes for water purification and microbial decontamination, which can be distributed to the target population especially the low lying regions prone to flooding in Kenya.

\section{Material and Methods}

2.1. Reagents and Instrumentation. All reagents were of analytical grade unless otherwise stated. The following reagents were obtained from Sigma-Aldrich Germany: 4,4' oxidianiline (ODA), pyromellitic dianhydride (PMDA), silver nitrate $\left(\mathrm{AgNO}_{3}\right)$, gold (III) chloride $\left(\mathrm{AuCl}_{3}\right)$, dimethyl formamide (DMF) and N,N-dimethyl acetamide (DMAc), and glass slides. All water used was triply distilled deionized water with a resistivity of $18 \mathrm{M} \Omega$ or better. Gold (III) chloride was dissolved in water to make a $0.1 \mathrm{M}$ aqueous solution. Thermal curing was achieved using a Thermo Scientific Isotemp programmable vacuum oven (series 750 , model 126). All samples were preheated to $35^{\circ} \mathrm{C}$ for $1 \mathrm{~h}$ before final thermal curing at $100^{\circ} \mathrm{C}$.

2.2. Synthesis of Poly (amic) Acid (PAA). The purified ODA was dissolved in the DMF/DMAc cosolvent to a concentration of $5 \mathrm{w} / \mathrm{w} \%$. PMDA was added to DMF and dissolved in the DMF/DMAc cosolvent. PMDA powder was dissolved in DMAc, after having precipitated from the dissolved lump material. The cosolvent solution was stirred at room temperature for 24 hours.

2.3. Incorporation of Gold Nanoparticles (AuNPs) into the $P A A . \mathrm{AuCl}_{3}$ was dissolved in $\mathrm{DMF}$ and $0.21 \mathrm{M}$ polyamic acid (PAA) solution was added. The reaction mixture was observed at room temperature with a solution of $\mathrm{AuCl}_{3}$ in DMF used as control. The setup was monitored over time using UV-vis spectroscopy to characterize the formation of AuNPs.

2.4. Incorporation of Silver Nanoparticles (AgNPs) into the $P A A .6 \mathrm{mg}$ of $\mathrm{AgNO}_{3}$ was dissolved in $5 \mathrm{~mL}$ of $\mathrm{DMF}$ and $0.21 \mathrm{M}$ PAA solution was added. The reaction mixture was monitored at room temperature at $70^{\circ} \mathrm{C}$ and $100^{\circ} \mathrm{C}$ with a 
solution of $\mathrm{AgNO}_{3}$ in DMF used as control. The setup was monitored over time and UV-vis spectroscopy was used to characterize the formation of AgNPs.

2.5. Simultaneous Incorporation of Au/AgNP-PAA Films. $20 \mathrm{ml}$ DMF was added to $28 \mu \mathrm{L}$ PAA and $5 \mathrm{mg} \mathrm{AgNO}_{3}$ was added. The solution was then monitored for 55 minutes at $100^{\circ} \mathrm{C}$. After 55 minutes, the solution was cooled, then $2.8 \mu \mathrm{L}$ $\mathrm{AuCl}_{3}$ was added into solution and further time evolution monitoring was done with UV-vis spectrophotometry.

2.6. Characterization of the Synthesized Nanoparticles. $\mathrm{UV}$-vis spectra of the synthesized Au and Ag nanoparticles were recorded at different times using a UV-vis spectrophotometer from $200 \mathrm{~nm}$ to $700 \mathrm{~nm}$.

2.7. Fabrication of the Nanostructured Polyamic Acid (nPAA) Membranes. After the silver and gold nanoparticles were incorporated in the PAA in solution, wet phase inversion process was employed to create the nanostructured PAA (nPAA) membranes. Viscous solutions of $10 \mathrm{w} / \mathrm{w} \%$ PAA, Ag-PAA, and Ag/Au-PAA were cast onto glass plates to a thickness of $\sim 120 \mu \mathrm{m}$ and allowed to evaporate for $2-3$ seconds before immersion in coagulation bath of deionized water/toluene at room temperature as described in literature [30]. The resulting thin layer that was fabricated onto glass substrate was then immersed in water for about 10 minutes. This step enabled the complete dissolution of DMAc in water allowing the insoluble PAA copolymer to precipitate into a dark, opaque, and smooth film. After 10 minutes, the film easily peeled off from the glass, forming a stand-alone nPAA membrane and flushed with water for about $1 \mathrm{~min}$, then dried in air under room temperature.

2.8. Surface Morphology. Surface morphology evaluation was done using Scanning Electron Microscopy (SEM). The SEM analyses were performed on PAA and modified PAA films to determine the morphology of the films and to identify the nature and distribution of the metallic particles in PAA membranes.

2.9. The Effect of Ag-PAA/Ag-Au-PAA Membranes on Microbial Growth. Diffusion tests were used for antimicrobial examination of nPAA films. Control, PAA, Ag-PAA, and $\mathrm{Au} / \mathrm{Ag}$-PAA films were placed on plates of Muller Hinton agar onto which Escherichia coli and Streptococcus aureus cells had been inoculated. The plates were then kept at $37^{\circ} \mathrm{C}$ overnight. Antimicrobial action of Ag-nPAA and AunPAA was accessed separately, then synergetic effects of using both nanoparticles was done. The inoculum was prepared by growing test strain in Mueller Hinton broth medium to $\sim 10^{4}-10^{5}$ colony forming units. In addition, effect of silver nanoparticles on microbial growth was tested using a liquid phase nutrient broth. The broth was prepared by mixing $\mathrm{MgSO}_{4}, \mathrm{CaCl}_{2}, \mathrm{KHPO}_{4}, \mathrm{KHPO}_{4}$, $\left(\mathrm{NH}_{4}\right) \mathrm{NO}_{3}, \mathrm{FeCl}_{3}$, and sucrose in a litre of distilled water and sterilized in an autoclave. Flasks of the broth were inoculated with the bacteria and the concentrations of silver nanoparticles were maintained at $20 \mu \mathrm{g} \mathrm{ml}^{-1}$ for both bacteria. The flasks were kept for 24 hours in a shaker at $150 \mathrm{rpm}$. UV-vis spectrometry was used to observe Optical density after 24 hours at $540 \mathrm{~nm}$. Absorbance of the broth mixture was used to calculate bacterial concentration using a standard curve.

\section{Results and Discussion}

3.1. Characterization of $A g-n P A A / A u-n P A A / A u-A g-n P A A$ Nanocomposite Solutions. PAA is a conducting electroactive polymer and its properties can be tuned by manipulating the delocalized $\mathrm{p}$ electron system for chemical and electrocatalytic applications. PAA provides a means of generating nanocomposites containing monodispersed metal particles while retaining its physical and chemical properties [28]. Distinct optical and physical properties dependent upon size, shape, surface structure, and agglomeration state are a characteristic of nanoparticles [31]. Gold and Silver nanoparticles display Localized Surface Plasmon Resonance (LSPR), which is a collective oscillation of electrons in the conducting band of nanoparticles in resonance with a specific wavelength of incident light [32]. The LSPR of silver nanoparticles results in a strong absorbance in the visible region, which can be measured by UV-vis spectroscopy [32] . Yellow, high viscosity polyamic acid was used in the in situ synthesis of gold and silver nanoparticles as described in the procedure. Dissolved poly (amic) acid and silver nitrate in 4 : 1 dimethyl formamide: dimethyl acetamide, changed color from clear to pale yellow, deep yellow, light reddish brown, and finally deep brown after 55 minutes at $100^{\circ} \mathrm{C}$ as shown in Figure 1 below.

Nanoparticles will absorb different frequencies of visible light by function of their size and reflect frequencies visible in the color shade of the solution. The color change was due to the different states of the LSPR of the silver nanoparticles at their characteristic wavelength, which was registered by the UV-vis absorption spectra peaks at $415 \mathrm{~nm}$ as shown in Figure 2(b) $[29,33]$ confirming the formation of the silver nanoparticles. Under room temperature, observable color change was not seen until after five days. In comparison, just five minutes was needed to see a change in color at $100^{\circ} \mathrm{C}$. Temperature thus affects the rate of nanoparticle formation. The solutions were also without precipitation even after 2 weeks indicating favorable homogeneity and good stabilization from agglomeration by the PAA medium. The UV-vis bands seen for silver nanoparticles were broad suggesting the nanoparticle's size polydispersivity. The intensity of peaks increased with time, indicating an increase in formation of the nanoparticles with temperature. At $100^{\circ} \mathrm{C}$ the Surface plasmon intensity of the reaction at 35 minutes was near that at 45 minutes signifying the completion of the reaction. Due to the sharp extinction peak at $\sim 415 \mathrm{~nm}$, the silver nanoparticles were ascribed an average size $30 \mathrm{~nm}$ by Lorentzian absorption profiles correlation tables [34]. 


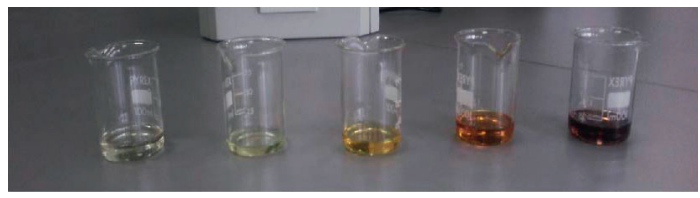

Figure 1: Color Changes in Ag-nPAA composite solution.

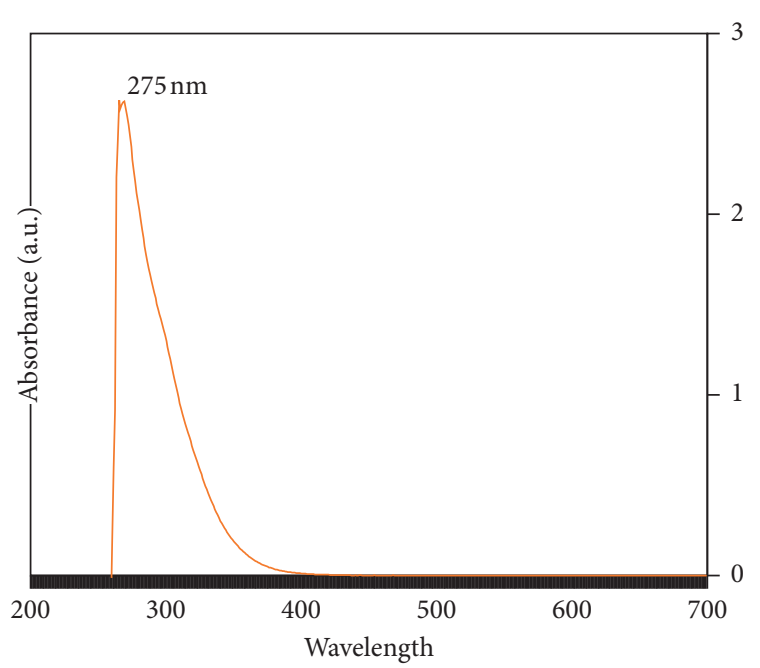

(a)

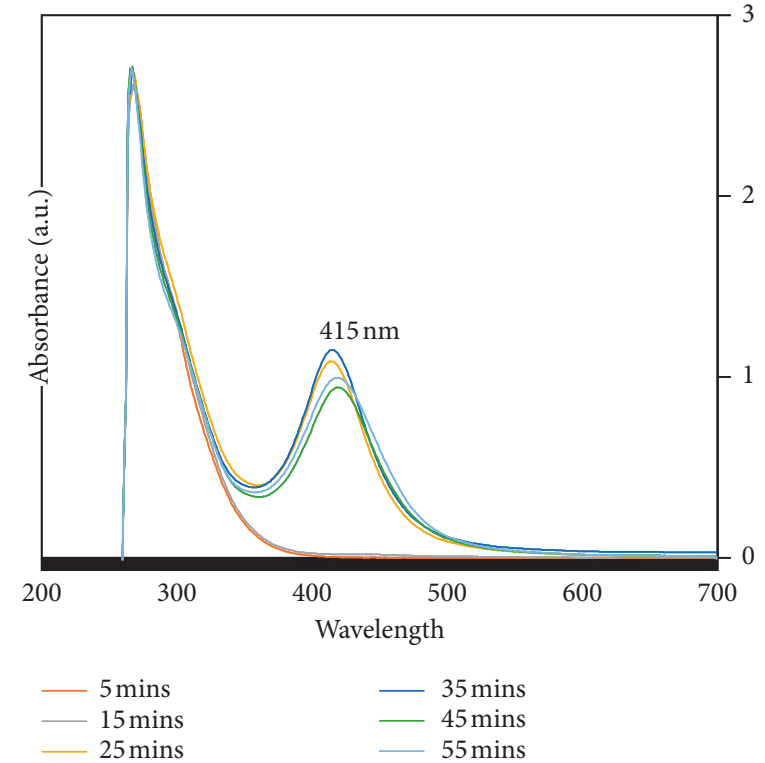

(b)

Figure 2: Time evolution of UV-vis absorption spectra of reaction mixture. (a) PAA spectrum (b) AgNPs at $100^{\circ} \mathrm{C}$.

3.1.1. Gold Nanoparticle Formation. $\mathrm{AuCI}_{3} / \mathrm{PAA}$ solution systems changed color from colorless to purple at room temperature. Color change of the solution and temporal growth of peaks at $540 \mathrm{~nm}$ indicated gold's nanoparticle formation (Figure 3(a)) [35]. Control experiments showed no change in color of suspension where PAA was absent. No absorption bands appear in the characteristic 500-600 nm region for gold nanoparticles formation when Gold chloride is dissolved in DMF even when monitored over an 80minute period. The color change is thus correlated to AuNPs. Size/shape dependence of Local Surface Plasmon Resonance spectrum of plasmonic nanoparticles was used to infer the size of synthesized particles to be $50-60 \mathrm{~nm}$ [36-38]. Considering that, upon binding of ligands to gold nanoparticles surface, the LSPR spectrum will red-shift by a few nanometers due to an increase in the local refractive index at the nanoparticles surface. At $100^{\circ} \mathrm{C}$, experiments witnessed nanoparticle aggregation at times beyond twenty minutes. Aggregation states were accompanied by a red shift in the spectrum, a broadening of absorption peaks, and a decrease in peak intensities as shown in Figure 3(b). Long temperature treatments thus had adversarial effects on the stability of gold nanoparticles. The aggregation of the particles is grossly undesirable in maintaining their catalytic ability. It was found that incorporation of silver nanoparticles at $100^{\circ} \mathrm{C}$ treatments, sudden cooling, and gold incorporation at ambient temperature achieved the best results in making highly efficient membranes.

3.1.2. Simultaneous Incorporation of $\mathrm{Ag}^{\mathrm{O}}$ and $\mathrm{Au} u^{\mathrm{O}}$ on Polyamic Acid. Silver forms as indicated increasing intensities of peaks at $\sim 415 \mathrm{~nm}$ over 55 minutes after which completion of reaction is observed. Upon addition of $\mathrm{AuCI}_{3}$ the solution immediately changed color from brick reddish brown to a pale grey color alluding to a competitive interaction between $\mathrm{Au}^{3+}$ and $\mathrm{Ag}^{+}$ions on PAA. Amide and carboxylic functionalities on the surface Polyamic acid interact with other species via loss of $\mathrm{H}^{+}$[39]. Gold and silver are less reactive than $\mathrm{H}^{+}$. These species are readily reduced by amide/carboxylic acid functionalities forming nanoparticles. In addition, $\mathrm{Au}^{+}$is more readily reduced to $\mathrm{Au}^{0}$ than $\mathrm{Ag}^{+}$. Gold thus replaces silver nanoparticles previously sequestered on PAA assuming total surface coverage. Incorporation of gold and silver nanoparticles thus requires careful considerations be made on the order and proportion of $\mathrm{Ag}$ and $\mathrm{Au}$ salts added to the PAA solution systems. Results seen in UV-vis time evolution demonstrate peak growth at $\sim 520 \mathrm{~nm}$ indicative of gold nanoparticle formation on silver-polyamic acid frameworks as illustrated in (Figure 4). This examination shows the possibility of simultaneous incorporation of AgNPs \& AuNPs in PAA frameworks. 


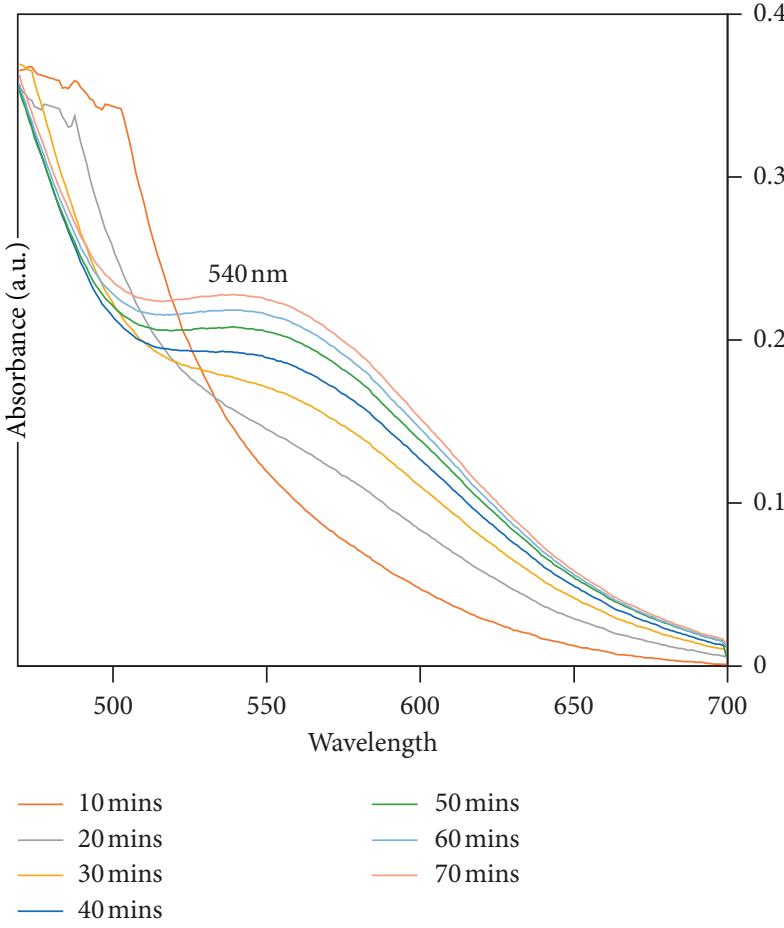

(a)

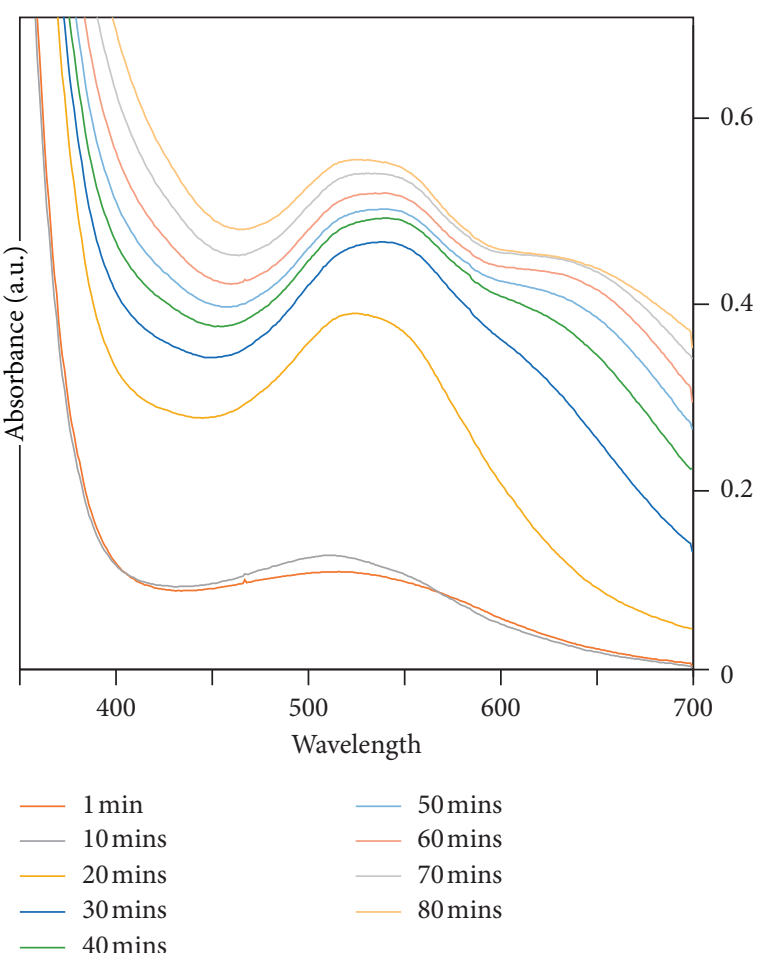

(b)

Figure 3: Time evolution of UV-vis absorption spectra of reaction mixture. (a) AuNPs at room temperature (b) AuNPs at $100^{\circ} \mathrm{C}$.

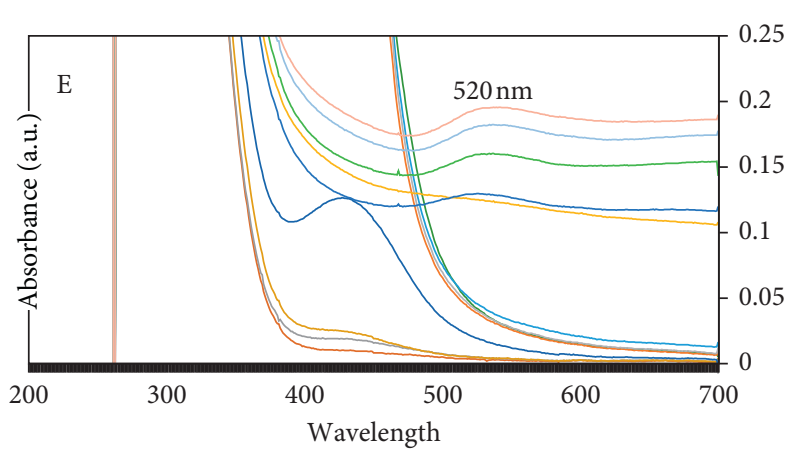

Figure 4: AgNPs at $100^{\circ} \mathrm{C}$ for 55 minutes, then addition of AuNPs at room temperature to 95 minute.

3.2. Fabrication of the Nanostructured Polyamic Acid Membrane. Membranes casts within deionized water were fragile to handle and are not pictured in Figure 5 below. Low molecular weight PAA formed fragile, highly porous membranes. Higher molecular weight solutions yielded increasingly stronger, more flexible membranes and are shown in (Figures 5(a)-5(c)). The wet phase inversion process used produced highly porous, strong and flexible nanostructured PAA (nPAA) membranes, which showed well-dispersed Gold and Silver nanoparticles. The pores on the surface of the membrane varied from 0.2 to $0.7 \mu \mathrm{m}$.

3.3. Surface Morphology. Polyamic acid and modified Polyamic acid films were subjected to Scanning Electron Microscopy for morphological determinations and for identification of the nature and distribution of metallic particles within them (Figures 6 and 7). Figure 6 shows the surface of wet phase inverted $\mathrm{Au} / \mathrm{Ag}$-PAA, with a spongelike morphology typically observed in asymmetric, phase inverted membranes described in literature [40, 41]. Gold and Silver nanoparticles are well dispersed on the film with an average size of $35 \mathrm{~nm}$ for spherical gold nanoparticles and $25 \mathrm{~nm}$ for Silver nanoparticles. Surface pores on the film varied from 0.2 to $0.7 \mu \mathrm{m}$.

The morphology of PAA films with a thermal curing step before phase inversion is noticeably different from that of the $\mathrm{Au} / \mathrm{Ag}-\mathrm{PAA}(\mathrm{w})$ film. Shown in Figure 7(a) is the image of a thermally cured phase inverted PAA film, and Figure $7(\mathrm{~b})$ shows an image of a thermally cured phase inverted Ag-PAA film. The surfaces of the PAA and Ag-PAA films were smooth and without any pores. Gold nanoparticles ranging from 30 to $120 \mathrm{~nm}$ are well dispersed on the surface. Most gold nanoparticles are spherical, and some larger nanoparticles have an elongated shape. Fewer gold and silver nanoparticles were observed on the surface of thermally cured phase inverted films (Figure 7 (c) inset) than the surface nanoparticles on the wet phase inverted films (Figure 6).

3.4. Antimicrobial Analysis. Antimicrobial assays were conducted as reported in the procedure. Silver nanoparticles demonstrated inhibition for both E. coli and Staphylococcus aureus. The antiseptic mechanism of silver NPs is described by the formation of pits in the cell wall and accumulation of AgNPs in the membrane resulting in increased permeability 

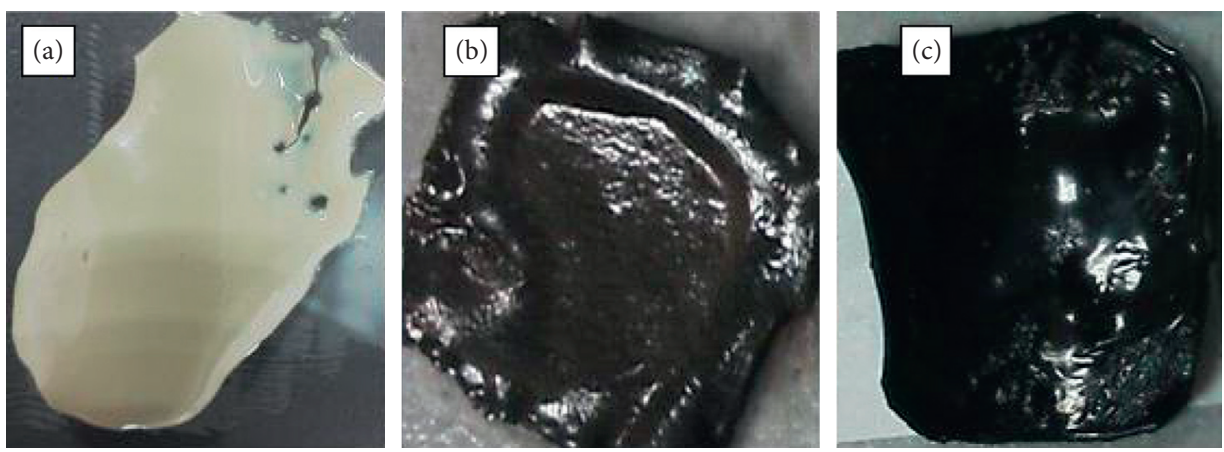

Figure 5: Knife cast wet phase inversion membranes of (a) Poly(amic) acid solution (b) Silver nanoassisted Poly(amic) acid membrane (c) Silver/Gold Nanoassisted Poly (amic) acid membrane. Actual sizes of pictured membranes are $3.5 \pm 0.5 \mathrm{~cm}$.

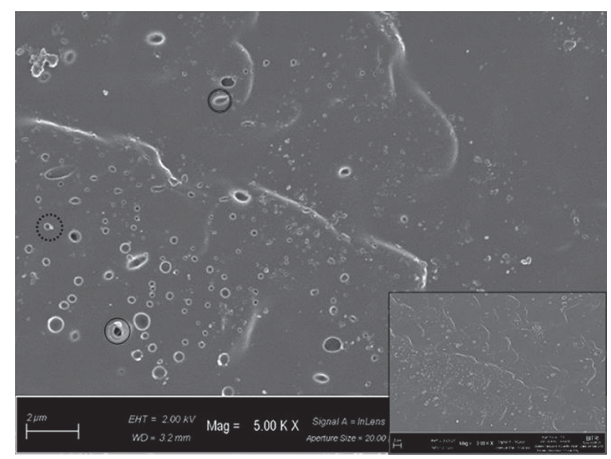

FIGURE 6: Scanning Electron Microscopy (SEM) image of wet phase inverted Au/Ag-PAA film. The main image shows the enlarged image of the solid circled part of the inset at a magnification of 5000x. The surface of the film shows well-dispersed gold (circles with a double line), silver nanoparticles (circles with a dashed line), and pores on the polymer surface (circles with a single line).

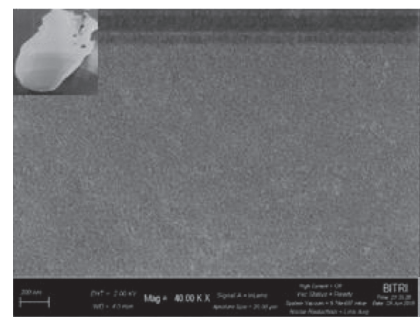

(a)

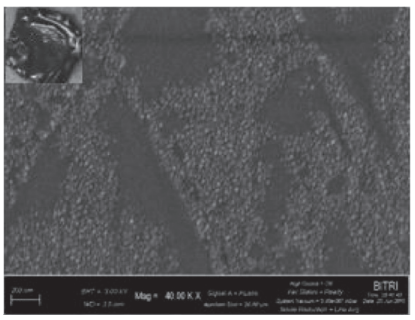

(b)

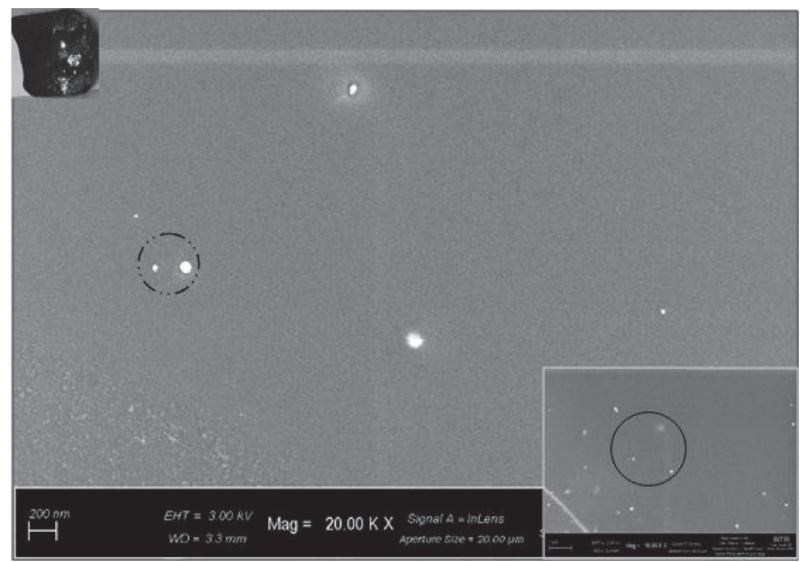

(c)

FIGURE 7: Scanning Electron Microscopy (SEM) images of thermally cured phase inverted PAA films: The insets are the recorded pictures of PAA films. (a) SEM image of the PAA film at magnifications of 40000x. (b) SEM image of the Ag-PAA film at magnifications of 40000x. Surface of the Ag-PAA film is with well-dispersed silver nanoparticles showing spherical shapes. (c) SEM image of the Au/Ag-PAA film at magnifications of 20000x. Surface of the Au/Ag-PAA film is with different nanoparticles. The circled part shows gold and silver nanoparticles in close proximity.

and inducing death in bacterial cells [42]. In Feng's reporting [43], the cytoplasmic membrane detaches from the cell wall. Cell TEM imaging indicates a mechanism showing interactions of silver with the sulfur of compounds in the cytoplasm and loss of DNA's replication abilities [44, 45]. A greater zone of inhibition with both bacteria was seen for $\mathrm{Au} / \mathrm{AgNPs}$ than when AgNPs alone were used suggesting a synergism between gold and silver nanoparticles (Figures 8 


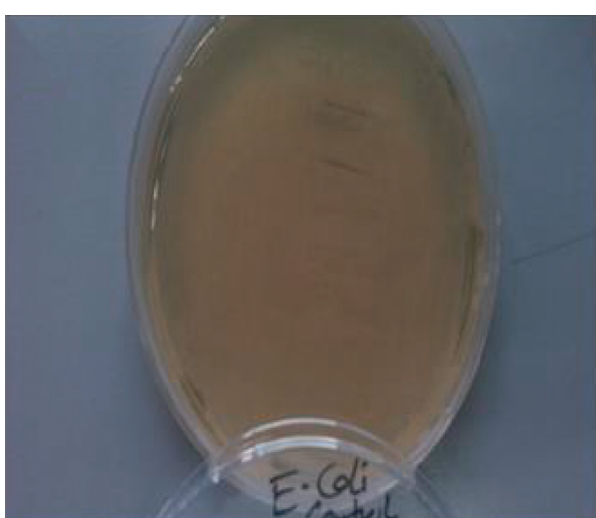

(a)

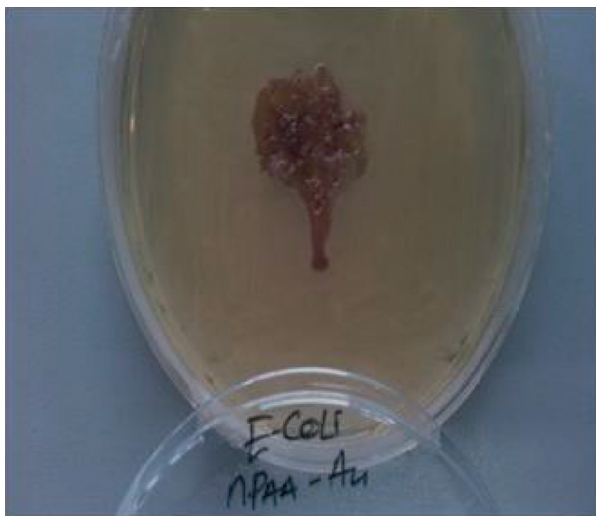

(c)

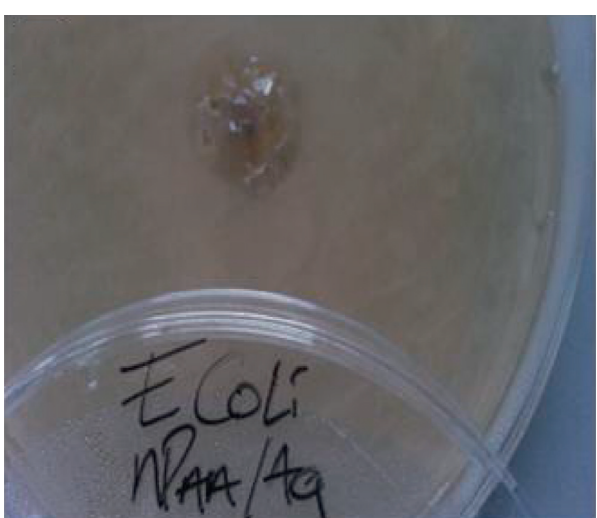

(b)

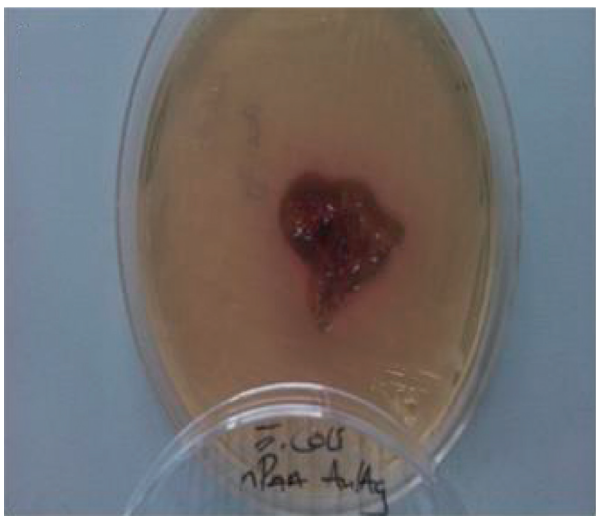

(d)

FIGURE 8: Investigation of the antimicrobial activity of different PAA solutions against E. coli. (a) Control plate with Muller Hinton agar and inoculated E. coli bacteria. (b) Plate treated with AuNPs-PAA. (c) plate treated with AgNPs-PAA. (d) Plate treated with Au/AgNPs-PAA demonstrating a larger zone of inhibition than the plate treated with AgNPs-PAA.

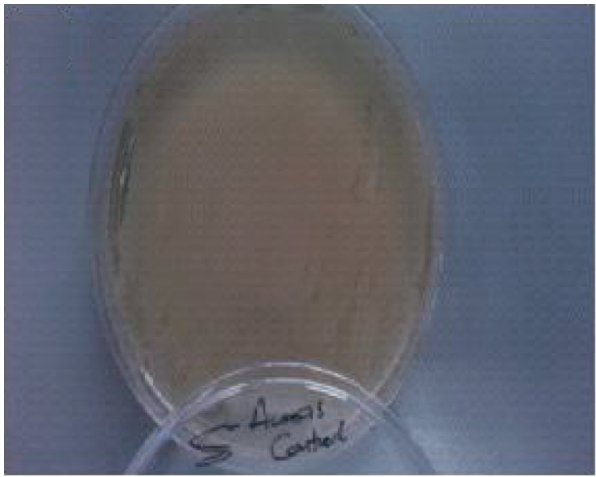

(a)

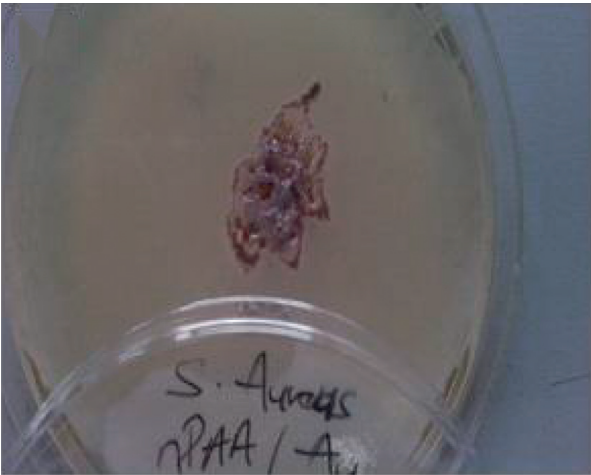

(b)

FIgURE 9: Continued. 


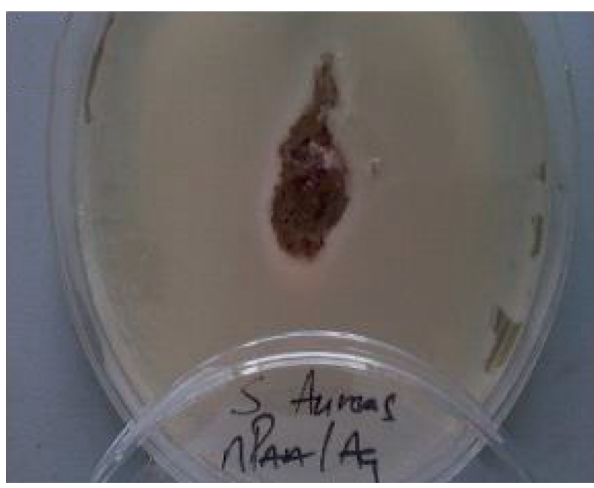

(c)

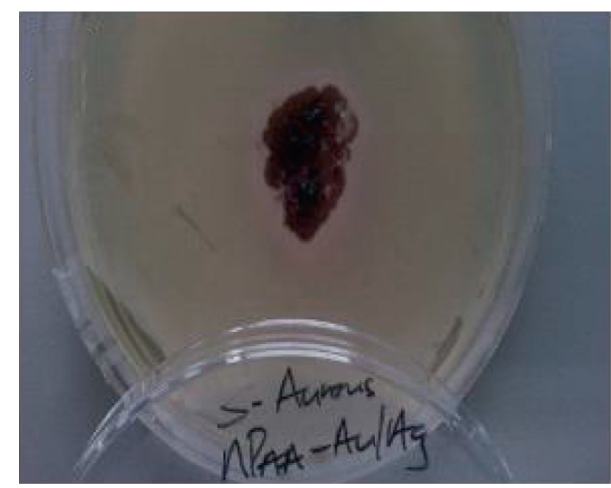

(d)

Figure 9: Investigation of the antimicrobial activity of different PAA solutions against S. aureus. (a) Control plate with Muller Hinton agar and inoculated S. aureus bacteria. (b) Plate treated with AuNPs-PAA (c) Plate treated with AgNPs-PAA (d) Plate treated with Au/AgNPsPAA demonstrating a larger zone of inhibition than the plate treated with AgNPs-PAA.

TABLE 1: UV-visible absorbance for broths treated with E. coli.

\begin{tabular}{lcc}
\hline & Absorbance/turbidity $(540 \mathrm{~nm})$ & Absorbance-(xi) \\
\hline Broth medium & 0.0417 & - \\
Sterilized broth medium with Au nanoparticles & 0.426 & - \\
Sterilized broth medium with Ag nanoparticles & 0.419 & - \\
Sterilized broth medium with $0.5: 0.5 \mathrm{Au} / \mathrm{Ag}$ nanoparticles & 0.429 & - \\
E. coli control & 0.221 & - \\
E. coli in PAA $: \mathrm{Au}$ & 0.694 & 0.182 \\
E. coli in PAA $: \mathrm{Ag}$ & 0.588 & 0.0845 \\
E. coli in PAA $: \mathrm{Au}: \mathrm{Ag}$ & 0.528 & 0.0238 \\
\hline
\end{tabular}

TABLE 2: UV-visible absorbance for broths treated with S. aureus.

\begin{tabular}{lcc}
\hline & Absorbance/turbidity $(540 \mathrm{~nm})$ & Absorbance due to the bacteria \\
\hline Broth medium & 0.0417 & - \\
Sterilized broth medium with Au nanoparticles & 0.426 & - \\
Sterilized broth medium with Ag nanoparticles & 0.419 & - \\
Sterilized broth medium with $0.5: 0.5$ Au/Ag nanoparticles & 0.429 & - \\
S. aureus control & 0.160 & - \\
S. aureus in PAA : Au & 0.554 & 0.128 \\
S. aureus in PAA : Ag & 0.489 & 0.0702 \\
S. aureus in PAA : Au : Ag & 0.438 & 0.0126 \\
\hline
\end{tabular}

and 9). Data obtained by UV-vis spectroscopy at $540 \mathrm{~nm}$ showed this enhancement by optical density measurements in liquid phase media as shown in Tables 1 and 2. Absorbance of the $E$. coli in suspension changed from 0.0845 with AgNPs alone to 0.0238 in the broth treated with PAA solution containing AuNPs and AgNPs indicating a better antimicrobial activity for combined Ag-AuNps. Gold nanoparticles by themselves depicted no antimicrobial activity for both E. coli and Staphylococcus aureus. This indicates that gold nanoparticles possess no antimicrobial properties as they are relatively inert but rather only enhance the biocidal properties of silver in the inhibition of microbial growth [46-48]. The enhancement of AuNPs in Staphylococcus aureus reduced the optical density Ag-AuNPs-PAA treated broth from 0.0702 to 0.0126 in the broth treated with Ag-PAA solution alone. This peculiar enhancement of
AgNPs action on cells observed with accompanying AuNPs could be due to the established catalytic character of gold and the widely studied gold-sulfur interface. The confluence of gold and silver forming sulfur bonds may be how mediation of silver NPs interaction with the sulfur of bacteria is achieved by the weak Au (0)-thiyl interface.

\section{Conclusion}

Reported in this paper is a two-step method for the simultaneous incorporation of well-dispersed gold and silver nanoparticles using PAA as both the reductant and sequestrating framework. Though with a degree of polydispersity, nanoparticles formed from silver nitrate had a narrow size distribution of $\sim 30 \mathrm{~nm}$ for AgNPs. AuNPs were affected by increased temperature regimes causing 
aggregation. The results demonstrated that modified PAA films have been successfully made through a wet phase inversion technique and the data obtained shows well-dispersed $\mathrm{Au}$ and Ag nanoparticles on the PAA polymer surface. Also demonstrated is a synergistic enhancement of AgNPs' antimicrobial activity by gold nanoparticles towards Gram positive (Staphylococcus aureus) and Gram negative (Escherichia coli) bacteria giving better antimicrobial material. The choice of coagulation of bath systems used in phase inversion of nanoassisted polymeric films is seen to affect the mechanical property of the polymer. Coagulation baths of high viscosity such as Toluene are preferred to solvents with high solvent/ nonsolvent exchange kinetics during phase inversion. This work demonstrates that the nanostructured polyamic acid can be used to remove microbials from water and can hence be used in water purification.

\section{Data Availability}

The data used to support the findings of this study are included within the article.

\section{Conflicts of Interest}

The authors declare that there are no conflicts of interest regarding the publication of this article.

\section{Acknowledgments}

The authors acknowledge United States International University-Africa (USIU-A) for research funds used for this work. Other than the funding and management of the funds, they had no other involvement in the study.

\section{References}

[1] Z. Wang, A. Wu, L. Colombi Ciacchi, and G. Wei, "Recent advances in nanoporous membranes for water purification," Nanomaterials, vol. 8, no. 2, p. 65, 2018.

[2] I. Tlili and T. A. Alkanhal, "Nanotechnology for water purification: electrospun nanofibrous membrane in water and wastewater treatment," Journal of Water Reuse and Desalination, vol. 9, no. 3, pp. 232-248, 2019.

[3] J. A. Camargo and Á. Alonso, "Ecological and toxicological effects of inorganic nitrogen pollution in aquatic ecosystems: a global assessment," Environment International, vol. 32, no. 6, pp. 831-849, 2006.

[4] L. A. Schaider, R. A. Rudel, J. M. Ackerman, S. C. Dunagan, and J. G. Brody, "Pharmaceuticals, perfluorosurfactants, and other organic wastewater compounds in public drinking water wells in a shallow sand and gravel aquifer," Science of The Total Environment, vol. 468-469, pp. 384-393, 2014.

[5] Neglected Diseases FAQ, "National Human Genome Research Institute," Neglected Diseases FAQ, Bethesda, MD, USA, https://www.genome.gov/FAQ/Neglected-Diseases.

[6] World Health Organization, United Nations Children's Fund, Progress on Sanitation and Drinking-Water, World Health Organization (WHO)/United Nations International Children's Fund (UNICEF) Joint Monitoring Programme for Water Supply and Sanitation, Geneva, Switzerland, 2010.

[7] Project, T.w. Health and Water. Improving Health in Africa, https://thewaterproject.org/why-water/health.
[8] J. Mansouri, S. Harrisson, and V. Chen, "Strategies for controlling biofouling in membrane filtration systems: challenges and opportunities," Journal of Materials Chemistry, vol. 20, no. 22, pp. 4567-4586, 2010.

[9] R. Li, L. Zhang, and P. Wang, "Rational design of nanomaterials for water treatment," Nanoscale, vol. 7, no. 41, pp. 17167-17194, 2015.

[10] R. P. Schwarzenbach, T. Egli, T. B. Hofstetter, U. von Gunten, and B. Wehrli, "Global water pollution and human Health," Annual Review of Environment and Resources, vol. 35, no. 1, pp. 109-136, 2010.

[11] C. Tang, Z. Wang, I. Petrinić, A. G. Fane, and C. HélixNielsen, "Biomimetic aquaporin membranes coming of age," Desalination, vol. 368, pp. 89-105, 2015.

[12] N. Ma, J. Wei, S. Qi, Y. Zhao, Y. Gao, and C. Y. Tang, "Nanocomposite substrates for controlling internal concentration polarization in forward osmosis membranes," Journal of Membrane Science, vol. 441, pp. 54-62, 2013.

[13] K. V. Lakshmi, N. Kriti, and J. Aditi, "Access to safe water: approaches for nanotechnology benefits to reach the bottom of the pyramid," May 2011.

[14] S. Andreescu, J. Njagi, C. Ispas, and M. T. Ravalli, "JEM Spotlight: applications of advanced nanomaterials for environmental monitoring," Journal of Environmental Monitoring, vol. 11, no. 1, pp. 27-40, 2009.

[15] I. Yazgan, N. Du, R. Congdon, V. Okello, and O. A. Sadik, "Biofunctionalized poly (amic) acid membranes for absolute disinfection of drinking water," Journal of Membrane Science, vol. 472, pp. 261-271, 2014.

[16] I. Pastoriza-Santos and L. M. Liz-Marzán, "Synthesis of silver nanoprisms in DMF," Nano Letters, vol. 2, no. 8, pp. 903-905, 2002.

[17] G. Lofrano, M. Carotenuto, G. Libralato et al., "Polymer functionalized nanocomposites for metals removal from water and wastewater: an overview," Water Research, vol. 92, pp. 22-37, 2016.

[18] T. K. Sau and C. J. Murphy, "Room temperature, high-yield synthesis of multiple shapes of gold nanoparticles in aqueous solution," Journal of the American Chemical Society, vol. 126, no. 28, pp. 8648-8649, 2004.

[19] R. A. Sperling, P. Rivera Gil, F. Zhang, M. Zanella, and W. J. Parak, "Biological applications of gold nanoparticles," Chemical Society Reviews, vol. 37, no. 9, pp. 1896-1908, 2008.

[20] N. Pandey, S. K. Shukla, and N. B. Singh, "Water purification by polymer nanocomposites: an overview," Nanocomposites, vol. 3, no. 2, pp. 47-66, 2017.

[21] S. Guo and E. Wang, "Synthesis and electrochemical applications of gold nanoparticles," Analytica Chimica Acta, vol. 598, no. 2, pp. 181-192, 2007.

[22] K. R. Shull and A. J. Kellock, "Metal particle adsorption and diffusion in a model polymer/metal composite system," Journal of Polymer Science Part B: Polymer Physics, vol. 33, no. 9, pp. 1417-1422, 1995.

[23] E. M. Vrijenhoek, S. Hong, and M. Elimelech, "Influence of membrane surface properties on initial rate of colloidal fouling of reverse osmosis and nanofiltration membranes," Journal of Membrane Science, vol. 188, no. 1, pp. 115-128, 2001.

[24] H.-L. Yang, J. C.-T. Lin, and C. Huang, "Application of nanosilver surface modification to RO membrane and spacer for mitigating biofouling in seawater desalination," Water Research, vol. 43, no. 15, pp. 3777-3786, 2009.

[25] H. A. Shawky, S.-R. Chae, S. Lin, and M. R. Wiesner, "Synthesis and characterization of a carbon nanotube/ 
polymer nanocomposite membrane for water treatment," Desalination, vol. 272, no. 1-3, pp. 46-50, 2011.

[26] S. Panigrahi, S. Basu, S. Praharaj et al., "Synthesis and sizeselective catalysis by supported gold nanoparticles: study on heterogeneous and homogeneous catalytic process," The Journal of Physical Chemistry C, vol. 111, no. 12, pp. 45964605, 2007.

[27] C. Batista, A. Lindomar, R. Caroline et al., "Nano-sized silver colloids produced and stabilized by amino-functionalized polymers: polymer structure-nanoparticle features and polymer structure-growth kinetics relationships," Journal of the Brazilian Chemical Society, vol. 28, no. 9, pp. 1608-1618, 2016.

[28] V. A. Okello, N. Du, B. Deng, and O. A. Sadik, "Environmental applications of poly (amic acid)-based nanomaterials," Journal of Environmental Monitoring, vol. 13, no. 5, pp. 1236-1245, 2011.

[29] V. Kariuki, Y. Idris, A. Ali, K. Andrzej, M. Parlinska-Wojtan, and S. Omowunmi, "Synthesis and catalytic, antimicrobial and cytotoxicity evaluation of gold and silver nanoparticles using biodegradable, П-conjugated polyamic acid," Environmental Science: Nano, vol. 2, 2015.

[30] S. Kim, K.-S. Jang, H.-D. Choi et al., "Porous polyimide membranes prepared by wet phase inversion for use in low dielectric applications," International Journal of Molecular Sciences, vol. 14, no. 5, pp. 8698-8707, 2013.

[31] I. Khan, K. Saeed, and I. Khan, "Nanoparticles: properties, applications and toxicities," Arabian Journal of Chemistry, vol. 12, no. 7, pp. 908-931, 2019.

[32] K. M. Kosuda, J. M. Bingham, K. L. Wustholz, R. P. Van Duyne, and R. J. Groarke, "4.06-nanostructures and surface-enhanced Raman Spectroscopy," in Comprehensive Nanoscience and Nanotechnology, D. L. Andrews, R. H. Lipson, and T. Nann, Eds., pp. 117-152, Academic Press, Cambridge, MA, USA, 2nd edition, 2016.

[33] A. Chhatre, P. Solasa, S. Sakle, R. Thaokar, and A. Mehra, "Color and surface plasmon effects in nanoparticle systems: case of silver nanoparticles prepared by microemulsion route," Colloids and Surfaces A: Physicochemical and Engineering Aspects, vol. 404, pp. 83-92, 2012.

[34] S. Agnihotri, S. Mukherji, and S. Mukherji, "Size-controlled silver nanoparticles synthesized over the range 5-100 nm using the same protocol and their antibacterial efficacy," RSC Advances, vol. 4, no. 8, pp. 3974-3983, 2014.

[35] N. N. Long, V. V. Le, D. K. Chu et al., "Synthesis and optical properties of colloidal gold nanoparticles," Journal of Physics: Conference Series, vol. 187, Article ID 012026, 2009.

[36] N. C. M. Tam, B. M. T. Scott, D. Voicu, B. C. Wilson, and G. Zheng, "Facile synthesis of Raman active phospholipid gold nanoparticles," Bioconjugate Chemistry, vol. 21, no. 12, pp. 2178-2182, 2010.

[37] R. E. Messersmith, G. J. Nusz, and S. M. Reed, "Using the localized surface plasmon resonance of gold nanoparticles to monitor lipid membrane assembly and protein binding," The Journal of Physical Chemistry C, vol. 117, no. 50, pp. 2672526733, 2013.

[38] E. T. Castellana, R. C. Gamez, and D. H. Russell, "Label-free biosensing with lipid-functionalized gold nanorods," Journal of the American Chemical Society, vol. 133, no. 12, pp. 4182-4185, 2011.

[39] T.-H. Young and L.-W. Chen, "Pore formation mechanism of membranes from phase inversion process," Desalination, vol. 103, no. 3, pp. 233-247, 1995.
[40] S. Qi, Z. Wu, D. Wu, W. Yang, and R. Jin, "The chemistry involved in the loading of silver(I) into poly (amic acid) via ion exchange: a metal-ion-induced crosslinking behavior," Polymer, vol. 50, no. 3, pp. 845-854, 2009.

[41] A. Idris, Z. Man, A. Maulud, and M. Khan, "Effects of phase separation behavior on morphology and performance of polycarbonate membranes," Membranes, vol. 7, no. 2, p. 21, 2017.

[42] I. Sondi and B. Salopek-Sondi, "Silver nanoparticles as antimicrobial agent: a case study on E. coli as a model for gramnegative bacteria," Journal of Colloid and Interface Science, vol. 275, no. 1, pp. 177-182, 2004.

[43] Q. L. Feng, J. Wu, G. Q. Chen et al., "A mechanistic study of the antibacterial effect of silver ions on Escherichia coli and Staphylococcus aureus," Journal of Biomedical Materials Research, vol. 52, no. 4, pp. 662-668, 2000.

[44] G. Han, P. Ghosh, M. De, and V. M. Rotello, "Drug and gene delivery using gold nanoparticles," NanoBiotechnology, vol. 3, no. 1, pp. 40-45, 2007.

[45] J. Conde, A. Ambrosone, V. Sanz et al., "Design of multifunctional gold nanoparticles for in vitro and in vivo gene silencing," ACS Nano, vol. 6, no. 9, pp. 8316-8324, 2012.

[46] L. Pezzi, A. Pane, F. Annesi et al., "Antimicrobial effects of chemically functionalized and/or photo-heated nanoparticles," Materials, vol. 12, no. 7, p. 1078, 2019.

[47] H. Gu, P. L. Ho, E. Tong, L. Wang, and B. Xu, "Presenting vancomycin on nanoparticles to enhance antimicrobial activities," Nano Letters, vol. 3, no. 9, pp. 1261-1263, 2003.

[48] E. Morales-Avila, G. Ferro-Flores, B. E. Ocampo-García et al., "Antibacterial efficacy of gold and silver nanoparticles functionalized with the ubiquicidin (29-41) antimicrobial peptide," Journal of Nanomaterials, vol. 2017, Article ID 5831959, 10 pages, 2017. 